\title{
STRUCTURE AND REPRESENTATIONS OF NONCOMMUTATIVE JORDAN ALGEBRAS
}

\author{
BY \\ KEVIN MCCRIMMON(1)
}

1. Introduction. The first three sections of this paper are devoted to proving an analogue of N. Jacobson's Coordinatization Theorem [6], [7] for noncommutative Jordan algebras with $n \geqq 3$ "connected" idempotents which characterizes such algebras as commutative Jordan algebras $\mathfrak{H}\left(\mathfrak{D}_{n}, \gamma\right)$ or split quasiassociative algebras $\mathfrak{D}_{n}^{(\lambda)}$. The proof proceeds by using certain deformations of the algebra and the base field which reduce the theorem to the special cases when the "indicator" $\phi$ is $\frac{1}{4}$ or 0 , and then using the Peirce decomposition to show that in these cases the algebra is commutative or associative respectively.

Next we develop the standard structure theory, using the Coordinatization Theorem to classify the simple algebras. The usual approach of A. A. Albert [2], [3] and R. H. Oehmke [9] proceeds by constructing an admissable trace function on the algebra $\mathfrak{A}$ to show that $\mathfrak{A}^{+}$is a simple Jordan algebra, and then characterizing the possible multiplications in $\mathfrak{A}$ yielding a given such $\mathfrak{A}^{+}$. Our approach is in some sense more intrinsic, and has the advantage of dealing simultaneously with all characteristics different from 2 ; the results for characteristic 3 seem to be new.

Another advantage of using the Coordinatization Theorem is that it furnishes a representation theory (in the sense of Eilenberg [4]) for the class of noncommutative Jordan algebras. The usual theorems hold only for birepresentations involving algebras of degree $\geqq 3$; counterexamples are given to show they fail spectacularly for algebras of degree 1 or 2 .

In this paper $\mathfrak{A}$ will always denote a noncommutative Jordan algebra (not necessarily finite-dimensional) over a field $\Phi$ of characteristic $\neq 2$. Thus for each $x \in \mathfrak{A}$ the linear transformations $L_{x}, R_{x}, L_{x^{2}}, R_{x^{2}}$ commute where $L_{y}, R_{y}$ denote the left and right multiplications by the element $y$. In linearized form

$$
\begin{aligned}
{\left[E_{x}, F_{y}\right]+\left[E_{y}, F_{x}\right]=0 } & (E, F=R, L) \\
{\left[E_{x y+y x}, F_{z}\right]+\left[E_{y z+z y}, F_{x}\right]+\left[E_{z x+x z}, F_{y}\right]=0 } & (E, F=R, L)
\end{aligned}
$$

Received by the editors November 23, 1964.

(1) This research was supported by a fellowship from the National Aeronautics and Space Administration and consists of part of the author's doctoral dissertation written at Yale University under Professor Nathan Jacobson. 
and these imply $[1, \mathrm{pp}$. 573-575]

$$
\begin{aligned}
L_{x y}-L_{x} L_{y} & =R_{y x}-R_{x} R_{y}, \\
L_{x} L_{y}\left(L_{x}+R_{x}\right)+R_{y x^{2}} & =R_{x^{2}} R_{y}+L_{x y}\left(L_{x}+R_{x}\right) .
\end{aligned}
$$

2. Peirce decompositions. We recall here the usual facts about Peirce decompositions [1, p. 591]. If $e \in \mathfrak{A}$ is an idempotent we have the vector-space direct sum

$$
\mathfrak{U}=\mathfrak{U}_{0} \oplus \mathfrak{U}_{1} \oplus \mathfrak{U}_{2}, \mathfrak{U}_{i}=\{x \mid e x+x e=i x\}
$$

and the associated projections $P_{i}$ on $\mathfrak{A}_{i}$; since these are polynomials in $L_{e}+R_{e}$, and since

$$
\left[E_{x}, F_{e}\right]=0 \quad \text { if } x \in \mathfrak{U}_{0}+\mathfrak{U}_{2}, \quad E, F=R, L,
$$

it follows that

$$
\left[E_{x}, P_{i}\right]=0 \text { if } x \in \mathfrak{A}_{0}+\mathfrak{A}_{2}, \quad E=R, L .
$$

The spaces satisfy

$$
\mathfrak{U}_{i}^{2} \subset \mathfrak{A}_{i}, \quad \mathfrak{A}_{i} \mathfrak{A}_{1}+\mathfrak{A}_{1} \mathfrak{A}_{i} \subset \mathfrak{U}_{1}, \quad x \in \mathfrak{U}_{i} \Rightarrow x e=e x=\frac{1}{2} i x \quad(i=0,2)
$$

$$
x, y \in \mathfrak{U}_{1} \Rightarrow x y+y x \in \mathfrak{U}_{0}+\mathfrak{U}_{2} .
$$

If $e=\sum_{i=1}^{n} e_{i}$ is the sum of orthogonal idempotents we have the Peirce decomposition

$$
\mathfrak{U}=\bigoplus_{i, j=0}^{n} \mathfrak{A}_{i j}
$$

where if all indices are distinct we have

$$
\begin{aligned}
& \mathfrak{U}_{00}=\{x \mid e x+x e=0\}, \quad \mathfrak{I}_{i i}=\left\{x \mid e_{i} x+x e_{i}=e x+x e=2 x\right\}, \\
& \mathfrak{U}_{i 0}=\left\{x \mid e_{i} x+x e_{i}=e x+x e=x\right\}=\mathfrak{A}_{0 i}, \\
& \mathfrak{U}_{i j}=\left\{x \mid e_{i} x+x e_{i}=e_{j} x+x e_{j}=x\right\}=\mathfrak{A}_{j i}, \\
& x \in \mathfrak{A}_{i j} \Rightarrow e_{i} x=x e_{j} \quad(\text { even if } i=j \neq 0) .
\end{aligned}
$$

We have the associated projection $P_{i j}$ on $\mathfrak{A}_{i j}$, and for distinct indices

$$
\mathfrak{U}_{i i}^{2} \subset \mathfrak{U}_{i i,}, \quad \mathfrak{A}_{i i} \mathfrak{A}_{i j}+\mathfrak{A}_{i j} \mathfrak{A}_{i i} \subset \mathfrak{U}_{i j},
$$

$$
\mathfrak{U}_{i j} \mathfrak{A}_{j k}+\mathfrak{A}_{j k} \mathfrak{A}_{i j} \subset \mathfrak{U}_{i k}, \quad \mathfrak{U}_{i j}^{2} \subset \mathfrak{U}_{i i}+\mathfrak{U}_{i j}+\mathfrak{U}_{j j}
$$


while all other products are zero. Note that $\mathfrak{U}_{00}=\mathfrak{A}_{i 0}=0$ if $e=1$ is the identity of $\mathfrak{A}$.

LEMMA 1. If $x, y \in \mathfrak{A}_{0}$ and $z, w \in \mathfrak{A}_{1}$ in (5) then

$$
\begin{aligned}
e(y z+z y) & =z y, \quad(y z+z y) e=y z, \\
P_{2}\{(e z) w+w(e z)\} & =P_{2}\{z w\}, \quad P_{0}\{(e z) w+w(e z)\}=P_{0}\{w z\}, \\
L_{x y} z & =L_{x} L_{y} z+L_{y} R_{x} z, \quad R_{x y} z=R_{x} L_{y} z+R_{y} R_{x} z .
\end{aligned}
$$

Proof. Replacing $x$ by $e$ in (3) shows $L_{e} L_{y}=R_{e} R_{y}$, so $e(y z)=L_{e} L_{y} z=R_{e} R_{y} z$ $=(z y) e=z y-e(z y) ;$ the second part of (10) follows by symmetry.

Next, $\quad P_{2}\{(e z) w+w(e z)\}=P_{2}\{(e z) w+w z-w(z e)\}=P_{2}\left\{w z+\left(L_{e z}-R_{z e}\right) w\right\}$ $=P_{2}\left\{w z+\left(L_{e} L_{z}-R_{e} R_{z}\right) w\right\}$ (by (3)) $=P_{2}\{z w\}$, and similarly for the other part of (11).

Replacing $y$ by $e$ in (2) gives $\left[R_{x z+z x}, L_{e}\right]+\left[R_{z}, L_{x}\right]=0$; allowing this to act on $y$, and noting $L_{e} y=0$, we obtain $R_{z} L_{x} y=L_{x} R_{z} y+L_{e} R_{x z+z x} y$. But $R_{z} L_{x} y$ $=(x y) z=L_{x y} z, \quad L_{x} R_{z} y=x(y z)=L_{x} L_{y} z, \quad$ and $\quad L_{e} R_{x z+z x} y=e\{y(x z+z x)\}$ $=y\{e(x z+z x)\}($ by $(6))=y(z x)$ (by (10)) $=L_{y} R_{x} z$. This yields the first relation in (12), and the second follows by symmetry.

LEMMA 2. If in (5) $\mathfrak{N}_{1} \subset \mathfrak{A}_{1}$ is a subspace with $\mathfrak{A}_{i} \mathfrak{N}_{1}+\mathfrak{N}_{1} \mathfrak{A}_{i} \subset \mathfrak{N}_{1}$ for $i=0,2$ then $\mathfrak{N}_{i}=P_{i}\left\{\mathfrak{A}_{1} \mathfrak{N}_{1}+\mathfrak{N}_{1} \mathfrak{A}_{1}\right\}$ is an ideal in $\mathfrak{A}_{i}$.

Proof. To prove $E_{x}\left\{\mathfrak{N}_{i}\right\} \subset \mathfrak{N}_{i}$ for $x \in \mathfrak{A}_{i}, E=R, L$ it suffices (by definition of $\mathfrak{N}_{i}$ ) to show $E_{x} P_{i} F_{y}\left\{\mathfrak{N}_{1}\right\} \subset \mathfrak{N}_{1}$ for $y \in \mathfrak{A}_{1}, F=R, L$. Putting $z=e$ in (2) gives $0=i\left[E_{x}, F_{y}\right]+\left[E_{y}, F_{x}\right]+\left[E_{w}, F_{e}\right]=(i-1)\left[E_{x}, F_{y}\right]+\left[E_{w}, F_{e}\right]$ using (1), where $w=x y+y x \in \mathfrak{A}_{1}$ by (7). Since $i-1 \neq 0, E_{x} P_{i} F_{y}\left\{\mathfrak{N}_{1}\right\}=P_{i} E_{x} F_{y}\left\{\mathfrak{N}_{1}\right\}$ (by (6)*) $\subset P_{i}\left\{F_{y} E_{x}\left\{\mathfrak{N}_{1}\right\}+E_{w} F_{e}\left\{\mathfrak{N}_{1}\right\}+F_{e} E_{w}\left\{\mathfrak{N}_{1}\right\}\right\} \subset P_{i}\left\{F_{y}\left\{\mathfrak{N}_{1}\right\}+E_{w}\left\{\mathfrak{N}_{1}\right\}\right\}+F_{e} P_{i}\left\{E_{w}\left\{\mathfrak{N}_{1}\right\}\right\}$ (by the assumption on $\left.\mathfrak{N}_{1}\right) \subset P_{i}\left\{F_{y}\left\{\mathfrak{N}_{1}\right\}\right\}+P_{i}\left\{E_{w}\left\{\mathfrak{N}_{1}\right\}\right\} \subset \mathfrak{N}_{i}$ as desired.

Corollary. If $\mathfrak{N}_{1}=\mathfrak{A}_{1}$ then $\mathfrak{B}=\mathfrak{N}_{0}+\mathfrak{N}_{1}+\mathfrak{N}_{2}$ is an ideal in $\mathfrak{A}$.

Proof. $\mathfrak{U N}_{1}+\mathfrak{N}_{1} \mathfrak{A} \subset \mathfrak{N}_{0}+\mathfrak{A}_{1}+\mathfrak{N}_{2}=\mathfrak{B}$ by definition of $\mathfrak{N}_{i}, \mathfrak{A}_{1}\left(\mathfrak{N}_{0}+\mathfrak{N}_{2}\right)$ $+\left(\mathfrak{N}_{0}+\mathfrak{N}_{2}\right) \mathfrak{A}_{1} \subset \mathfrak{A}_{1} \subset \mathfrak{B}$ by (7), and $\mathfrak{A}_{i}\left(\mathfrak{N}_{0}+\mathfrak{N}_{2}\right)+\left(\mathfrak{N}_{0}+\mathfrak{N}_{2}\right) \mathfrak{A}_{i} \subset \mathfrak{N}_{0}+\mathfrak{N}_{2}$ $\subset \mathfrak{B}$ if $i=0,2$ by (7) and the Lemma.

LEMma 3. If for $\lambda \in \Phi$ we define $\mathfrak{A}_{1}^{[\lambda]}=\left\{x \in \mathfrak{U}_{1} \mid L_{e} x=\lambda x\right\}$ then for $i=0,2$ we have $\mathfrak{A}_{i} \mathfrak{A}_{1}^{[\lambda]}+\mathfrak{U}_{1}^{[\lambda]} \mathfrak{U}_{i} \subset \mathfrak{A}_{1}^{[\lambda]}$.

Proof. If $y \in \mathfrak{A}_{i}$ then by (6) $L_{y}$ and $R_{y}$ commute with $L_{e}$, hence with $L_{e}-\lambda I$, so they leave $\operatorname{ker}\left(L_{e}-\lambda I\right)$ invariant; by (7) they leave $\mathfrak{A}_{1}$ invariant, so they leave invariant $\mathfrak{A}_{1} \cap \operatorname{ker}\left(L_{e}-\lambda I\right)=\mathfrak{A}_{1}^{[\lambda]}$.

3. Algebras with connected idempotents. If $\lambda \in \Phi$ and $e \in \mathfrak{A}$ is an idempotent then $\mathfrak{C}_{1}^{[\phi]}(e)=\mathfrak{U}_{1}^{[\lambda]}(e)+\mathfrak{I}_{1}^{[1-\lambda]}(e)$ is completely determined by $\phi=\lambda(1-\lambda)$; it 
can be thought of as the "eigenspace" of $L_{e}$ and $R_{e}$ corresponding to the "eigenvalue" $\phi$. In (8) we set $\mathfrak{E}_{i j}^{[\phi]}=\mathbb{E}_{1}^{[\phi]}\left(e_{i}\right) \cap \mathfrak{E}_{1}^{[\phi]}\left(e_{j}\right)$. Then we shall say $e_{i}$ and $e_{j}$ are connected if there is a $\phi \in \Phi$ and $u \in \mathfrak{E}_{i j}^{[\phi]}$ which is regular [6,p. 1156], [11, p. 19] in the subalgebra $\mathfrak{U}_{i i}+\mathfrak{U}_{i j}+\mathfrak{A}_{j j} ; \phi$ will be called an indicator of $\mathfrak{U}_{i j}$. In this case there is a $v$ with $u v=v u=e_{i}+e_{j}$, and $v=\left(u^{2}\right)^{-1} u \in\left(\mathfrak{A}_{i i}+\mathfrak{A}_{j j}\right) \mathfrak{E}_{i j}^{[\phi]} \subset \mathfrak{E}_{i j}^{[\phi]}$ by (7) and Lemma 3. In this section we assume $1=\sum e_{i}$ is the sum of $n \geqq 3$ connected orthogonal idempotents.

LEMMA 4. All indicators have a common value $\phi$, and we have the relations

$$
\begin{aligned}
\mathfrak{A}_{i j} & =\mathfrak{E}_{i j}^{[\phi]} & & (i \neq j), \\
\mathfrak{U}_{i j} & =\mathfrak{A}_{i k} \mathfrak{A}_{k j}+\mathfrak{A}_{k j} \mathfrak{A}_{i k} & & (i, j, k \neq), \\
\mathfrak{I}_{i i} & =P_{i i}\left\{\mathfrak{A}_{i k}^{2}\right\} & & (i \neq k), \\
\mathfrak{A}_{i k}^{2} & \subset \mathfrak{U}_{i i}+\mathfrak{U}_{k k} & & (i \neq k) .
\end{aligned}
$$

If $e=e_{k}$ then

$$
\begin{aligned}
\mathfrak{U}_{1} & =\mathfrak{F}_{1}^{[\phi]}, \\
\mathfrak{A}_{i} & =P_{i}\left\{\mathfrak{U}_{1}^{2}\right\} \quad(i=0,2), \\
\mathfrak{U}_{1}^{2} & \subset \mathfrak{U}_{0}+\mathfrak{U}_{2} .
\end{aligned}
$$

Proof. Given $i, j$ (not necessarily distinct) we can find $k \neq i, j$ by the assumption that $n \geqq 3$; take $e=e_{k}, \mathfrak{N}_{1}=\mathscr{C}_{1}^{\left[\phi_{i k}\right]}\left(e_{k}\right)$ in Lemma 2 where $\phi_{i k}$ is an indicator of $\mathfrak{A}_{i k}$. Then there are $u, v \in \mathfrak{E}_{i k}^{\left[\phi_{i k}\right]} \subset \mathfrak{N}_{1}$ with $e_{i}=P_{0}\left(e_{i}+e_{k}\right)=P_{0}(u v) \in \mathfrak{N}_{0}$, so by Lemma 2

$$
\mathfrak{A}_{i j}=e_{i} \mathfrak{A}_{i j}+\mathfrak{A}_{i j} e_{i} \subset \mathfrak{N}_{0} \mathfrak{A}_{0}+\mathfrak{A}_{0} \mathfrak{N}_{0} \subset \mathfrak{N}_{0}, \mathfrak{A}_{i j} \subset P_{i j}\left\{\mathfrak{N}_{0}\right\}=P_{i j}\left\{\mathfrak{A}_{1} \mathfrak{N}_{1}+\mathfrak{N}_{1} \mathfrak{A}_{1}\right\}
$$

Now $\mathfrak{U}_{1}\left(e_{k}\right)=\sum_{l \neq k} \mathfrak{A}_{k l}$ by $(8)^{*}$, thus by (9) we see $P_{i j}\left\{\mathfrak{A}_{1} \mathfrak{N}_{1}+\mathfrak{N}_{1} \mathfrak{A}_{1}\right\}$ $=P_{i j}\left\{\mathfrak{A}_{j k} \mathbb{E}_{i k}^{\left[\phi_{i k}\right]}+\mathbb{E}_{i k}^{\left[\phi_{i k}\right]} \mathfrak{A}_{j k}+\mathfrak{A}_{i k} \mathfrak{E}_{j k}^{\left[\phi_{i k}\right]}+\mathfrak{C}_{j k}^{\left[\phi_{i k}\right]} \mathfrak{A}_{i k}\right\}$. This proves

$$
\begin{aligned}
& \mathfrak{U}_{i j} \subset \mathfrak{U}_{i k} \mathfrak{A}_{k j}+\mathfrak{U}_{k j} \mathfrak{A}_{i k}, \\
& \mathfrak{U}_{i i} \subset P_{i i}\left\{\mathfrak{U}_{i k}^{2}\right\},
\end{aligned}
$$

and also that for $i, j, k$ distinct

$$
\mathfrak{A}_{i j} \subset \mathfrak{C}_{i j}^{\left[\phi_{i k}\right]}
$$

by Lemma 3. Thus $\phi_{i j}=\phi_{i k}$ for any distinct $i, j, k$ and any choice of indicators, so clearly they have a common value, say $\phi$. Then the previous formula becomes

$$
\mathfrak{U}_{i j} \subset \mathfrak{E}_{i j}^{[\phi]} \text {. }
$$

These establish (13), (14), (15). For (16) let $x, y \in \mathfrak{A}_{i k}, z \in \mathfrak{A}_{i j}(j \neq i, k)$. Putting 
$e=e_{j}$ in (12) we see that the right-hand sides are in $\mathfrak{A}_{i j}$ by (9), so $E_{z}(x y) \in \mathfrak{A}_{i j}$ for $E=R, L$. Then by (6)* with $e=e_{k} E_{z} P_{1}(x y)=P_{1} E_{z}(x y) \subset P_{1}\left\{\mathfrak{A}_{i j}\right\}=0$. If $w=P_{1}(x y)=P_{i k}(x y)$ then $z w=w z=0$ for all $z \in \mathfrak{A}_{i j}$. But by connectivity we can find $u, v \in \mathfrak{A}_{i j}$ with $u v=v u=e_{i}+e_{j}$, so by (12) $L_{e_{i}} w=R_{e_{i}} w=0, w=0$, and $x y \in \mathfrak{A}_{i i}+\mathfrak{U}_{k k}$. (13)* follows from (13) and the above characterization of $\mathfrak{A}_{1}\left(e_{k}\right)$, and similarly (14)* follows from (14) and (15), while (15)* follows from (14) and (16).

The $\phi \in \Phi$, which is uniquely determined by the algebra $\mathfrak{A}$, will be called the indicator of $\mathfrak{A}$.

LEMMA 5. If the indicator is $\phi=\frac{1}{4}$ then $\mathfrak{A}$ is commutative.

Proof. Let $\mathfrak{B}=\left\{x \mid L_{x}=R_{x}\right\}$; if $x, y \in \mathfrak{B}$ then by (3) $L_{x y}-R_{x y}=L_{x y}-R_{y x}$ $=L_{x} L_{y}-R_{x} R_{y}=L_{x} L_{y}-L_{x} L_{y}=0$, and $x y \in \mathfrak{B}$. Thus $\mathfrak{B}$ is a subalgebra of $\mathfrak{A}$. We must show $\mathfrak{B}=\mathfrak{A}$.

Now $\lambda(1-\lambda)=\frac{1}{4}$ implies $\lambda=1-\lambda=\frac{1}{2}$, so by (13)* $L_{e_{k}}=R_{e_{k}}=\frac{1}{2} I$ on $\mathfrak{A}_{1}\left(e_{k}\right)$, and by (7) this shows $L_{e_{k}}=R_{e_{k}}$, and $e_{k} \in \mathfrak{B}$. Hence $P_{i i}\{\mathfrak{B}\} \subset \mathfrak{B}$ since $P_{i i}$ is a polynomial in $L_{e_{i}}=R_{e_{i}}$. Since $\mathfrak{A}_{i i}=P_{i i}\left\{\mathfrak{A}_{i k}^{2}\right\}$ by (15) it will be enough to prove $\mathfrak{A}_{i k} \subset \mathfrak{B}$ for $i \neq k$.

If $y \in \mathfrak{A}_{i}\left(e_{k}\right)(i=0,1,2)$ then from (3) with $x=e_{k}$ we get $\left(L_{e_{k}}-\frac{1}{2} i I\right)\left(L_{y}-R_{y}\right)=0$, so $\left(L_{y}-R_{y}\right) \mathfrak{A} \subset \mathfrak{A}_{i}$. Thus if $y \in \mathfrak{A}_{1}$ then $y^{2} \in \mathfrak{A}_{0}+\mathfrak{A}_{2}$ implies $\left(L_{y^{2}}-R_{y^{2}}\right) \mathfrak{U}$ $\subset \mathfrak{U}_{0}+\mathfrak{A}_{2}$; but from (3) with $x=y$ we get $\left(L_{y^{2}}-R_{y^{2}}\right) \mathfrak{U}=\left(L_{y^{2}}-R_{y^{2}}\right) \mathfrak{U}$ $=\left(L_{y}-R_{y}\right)\left(L_{y}+R_{y}\right) \mathfrak{A} \subset\left(L_{y}-R_{y}\right) \mathfrak{U} \subset \mathfrak{U}_{1}$, so $\left(L_{y^{2}}-R_{y^{2}}\right) \mathfrak{U}=0, L_{y^{2}}=R_{y^{2}}$, and $y^{2} \in \mathfrak{B}$.

Given $i \neq k$ there is $j \neq i, k$ by the assumption $n \geqq 3$. Let $x \in \mathfrak{U}_{i j}, \quad y \in \mathfrak{A}_{j k}$; then $x, y \in \mathfrak{A}_{1}\left(e_{j}\right)$ so $x y+y x \in \mathfrak{B}$ by the above. From (10) we see $x y$ $=e_{i}(x y+y x) \in \mathfrak{B}$, and then from (14) we get $\mathfrak{A}_{i k} \subset \mathfrak{B}$ as desired.

Lemma 6. If the indicator is $\phi=0$ then $\mathfrak{A}$ is associative.

Proof. $\lambda(1-\lambda)=\phi=0$ implies $\lambda=0$ or $\lambda=1$. Letting $e=e_{k}$, (13)* shows $\mathfrak{A}_{1}=\mathfrak{C}_{1}^{[0]}=\mathfrak{A}_{1}^{[0]}+\mathfrak{A}_{1}^{[1]}$. Taking $x=z, y=e$ in (3) gives

$$
\begin{aligned}
& R_{z}=0 \text { on } \mathfrak{U}_{1}^{[0]} \text { if } z \in \mathfrak{A}_{0}, \\
& L_{z}=0 \text { on } \mathfrak{U}_{1}^{[0]} \text { if } z \in \mathfrak{A}_{2}
\end{aligned}
$$

since $L_{e}=0, R_{e}=I$ on $\mathfrak{I}_{1}^{[0]}$; dual results hold on $\mathfrak{I}_{1}^{[1]}$.

We must show $\left[L_{x}, R_{y}\right]=0$ for all $x \in \mathfrak{A}_{i j}, y \in \mathfrak{A}_{k l} ;$ since $n \geqq 3$ we see by (8) that we can always find $e=e_{m}$ such that $x \in \mathfrak{A}_{0}, y \in \mathfrak{A}_{i}(i=0,1,2)$.

First consider $y \in \mathfrak{A}_{1}$. Putting $z=e$ in (2) shows $\left[R_{y}, L_{x}\right]=-\left[R_{y^{\prime}}, L_{e}\right]$ for $y^{\prime}=x y+y x \in \mathfrak{A}_{1}$; hence it suffices to show $\left[L_{e}, R_{y}\right]=0$, and by symmetry we may suppose $y \in \mathfrak{A}_{1}^{[0]}$. If $z \in \mathfrak{A}_{0}$ then $\left[L_{e}, R_{y}\right] z=L_{e} R_{y} z=L_{e} L_{z} y=L_{z} L_{e} y=0$ by (6) and the definition of $\mathfrak{U}_{1}^{[0]}$; if $z \in \mathfrak{A}_{2}$ then $\left[L_{e}, R_{y}\right] z=L_{e} R_{y} z-R_{y} z=\left(L_{e}-I\right) L_{z} y$ $=0$ by the second formula above; if $z \in \mathfrak{A}_{1}^{[0]}$ then $\left[L_{e}, R_{y}\right] z=L_{e} R_{y} z \subset L_{e}\left\{\mathfrak{A}_{1}^{2}\right\} \subset \mathfrak{A}_{2}$ 
by (15)*, and $\left[R_{e}, L_{y}\right] z=R_{e} L_{y} z-L_{y} z \in\left(R_{e}-I\right)\left\{\mathfrak{U}_{1}^{2}\right\} \subset \mathfrak{A}_{0}$, so from (1) $\left[L_{e}, R_{y}\right] z=\left[R_{e}, L_{y}\right] z \in \mathfrak{U}_{0} \cap \mathfrak{A}_{2}=0$; similarly $\left[L_{e}, R_{y}\right] z=0$ if $z \in \mathfrak{A}_{1}^{[1]}$. We have thus shown $\left[L_{e}, R_{y}\right] \mathfrak{A}=0$, i.e., $\left[L_{e}, R_{y}\right]=0$.

Next, consider $y \in \mathfrak{A}_{0}$. By (7) $\left[L_{x}, R_{y}\right] \mathfrak{A}_{2}=0$; by the first formula above and Lemma $3\left[L_{x}, R_{y}\right] \mathfrak{A}_{1}^{[0]}=-R_{y} L_{x} \mathfrak{A}_{1}^{[0]} \subset R_{y} \mathfrak{A}_{1}^{[0]}=0$, and dually $\left[L_{x}, R_{y}\right] \mathfrak{A}_{1}^{[1]}=0$. Now let $u, v \in \mathfrak{A}_{1}$; then $\left[L_{x}, R_{y}\right](u v)=x\{(u v) y\}-\{x(u v)\} y=x\{u(v y)\}-\{(x u) v\} y$ $=(x u)(v y)-(x u)(v y)=0$ by the previous case where $\left[L_{z}, R_{w}\right]=\left[L_{w}, R_{z}\right]=0$ for $z \in \mathfrak{A}_{0}, w \in \mathfrak{U}_{1}$ since $x, y \in \mathfrak{A}_{0}$ and $u, v, x u, v y \in \mathfrak{A}_{1}$. Then from (14)* and (6)* $\left[L_{x}, R_{y}\right] \mathfrak{U}_{0}=\left[L_{x}, R_{y}\right] P_{0}\left\{\mathfrak{U}_{1}^{2}\right\}=P_{0}\left[L_{x}, R_{y}\right]\left\{\mathfrak{A}_{1}^{2}\right\}$, and this last is zero by what we just proved. Hence again $\left[L_{x}, R_{y}\right] \mathfrak{U}=0$ implies $\left[L_{x}, R_{y}\right]=0$.

Finally, if $y \in \mathfrak{A}_{2}$ then putting $z=e_{k}$ in (2) yields $2\left[L_{x}, R_{y}\right]=0$.

4. Mutations. If $\mathfrak{A}=(\mathfrak{X}, \cdot)$ is an algebra on $\mathfrak{X}$ and $\lambda \in \Phi$ then the $\lambda$-mutation of $\mathfrak{U}$ is the algebra $\mathfrak{U}^{(\lambda)}=\left(\mathfrak{X}, \lambda_{\lambda}\right)$ where

$$
x \cdot{ }_{\lambda} y=\lambda x \cdot y+(1-\lambda) y \cdot x .
$$

Note that $\mathfrak{A}^{(1 / 2)}$ is just the symmetrized algebra $\mathfrak{A}^{+}$.

The map $T: \lambda \rightarrow(\lambda+1) / 2$ is a $1-1$ map of $\Phi$ onto itself with inverse $T^{-1}: \lambda \rightarrow 2 \lambda-1$, so in a natural way it carries the field $\Phi=(\Phi,+, \cdot)$ isomor phically onto a field $\tilde{\Phi}=(\Phi, \oplus, \odot)$ where

$$
\begin{aligned}
& \lambda \oplus \mu=T\left(T^{-1} \lambda+T^{-1} \mu\right)=T(2 \lambda+2 \mu-2)=\lambda+\mu-\frac{1}{2}, \\
& \lambda \odot \mu=T\left(T^{-1} \lambda \cdot T^{-1} \mu\right)=T(4 \lambda \mu-2 \lambda-2 \mu+1)=2 \lambda \mu-\lambda-\mu+1 .
\end{aligned}
$$

$\tilde{\Phi}$ has zero element $T(0)=\frac{1}{2}$ and identity $T(1)=1$.

Consider the double mutation $\left\{\mathfrak{U}^{(\lambda)}\right\}^{(\mu)}$; multiplication is given by

$$
\begin{aligned}
x \cdot{ }_{\lambda, \mu} y & =\mu x \cdot{ }_{\lambda} y+(1-\mu) y \cdot{ }_{\lambda} x \\
& =\{\mu \lambda+(1-\mu)(1-\lambda)\} x \cdot y+\{\mu(1-\lambda)+\lambda(1-\mu)\} y \cdot x \\
& =\{\lambda \odot \mu\} x \cdot y+\{1-\lambda \odot \mu\} y \cdot x
\end{aligned}
$$

and so

$$
\mathfrak{U}^{(\lambda)(\mu)}=\mathfrak{U}^{(\lambda \cdot \mu)}
$$

If $\lambda \neq \frac{1}{2}$ then $\lambda$ has an inverse $\mu$ in $\tilde{\Phi}$, so we can recover $\mathfrak{A}$ from $\mathfrak{I}^{(\lambda)}$ : $\mathfrak{U}=\mathfrak{A}^{(1)}=\mathfrak{A}^{(\lambda \cdot \mu)}=\left\{\mathfrak{U}^{(\lambda)}\right\}^{(\mu)}$. However, if $\lambda=\frac{1}{2}$ we cannot recover $\mathfrak{A}$ because all mutations have the same $\mathfrak{I}^{+}: \mathfrak{I}^{(1 / 2)(\mu)}=\mathfrak{U}^{(\mu)(1 / 2)}=\mathfrak{A}^{(\mu \cdot 1 / 2)}=\mathfrak{U}^{(1 / 2)}$. Thus we cannot so easily recover an associative algebra $\mathfrak{A}$ from the special Jordan algebra $\mathfrak{A}^{+}$.

Note that an ideal in $\mathfrak{A}$ remains an ideal in $\mathfrak{I}^{(\lambda)}$, so if $\lambda \neq \frac{1}{2}$ ideals in $\mathfrak{A}$ and $\mathfrak{U}^{(\lambda)}$ coincide. Since $L_{x}^{(\lambda)}=\lambda L_{x}+(1-\lambda) R_{x}, R_{x}^{(\lambda)}=\lambda R_{x}+(1-\lambda) L_{x}$ it is clear that a mutation of a noncommutative Jordan algebra is again a noncommutative Jordan algebra. 
As an example, a split quasi-associative algebra is a mutation $\mathfrak{D}^{(\lambda)}$ of an associative algebra $\mathfrak{D}$. Then $\mathfrak{A}$ is quasi-associative if there is an extension $\Omega$ of $\Phi$ such that $\mathfrak{A}_{\Omega}$ is a split quasi-associative algebra over $\Omega: \mathfrak{U}_{\Omega}=\mathfrak{D}^{(\lambda)}$ for $\lambda \in \Omega$. $\mathfrak{A}$ is split if and only if $\lambda \in \Phi$. It is known that the indicator $\phi=\lambda(1-\lambda)$ of $\mathfrak{A}$ is always in $\Phi$, so it suffices to consider only quadratic extensions $\Omega=\Phi(\lambda)$.

These results were first obtained by A. A. Albert [1, pp. 581-584] although he did not make explicit use of $\tilde{\Phi}$.

TheOREM 1 (COORDINATIZATION THEOREM). If $\mathfrak{A}$ is a noncommutative Jordan algebra with $n \geqq 3$ connected orthogonal idempotents then either $\mathfrak{U}=\mathfrak{D}_{n}^{(\lambda)}$ is a split quasi-associative algebra determined by the algebra $\mathfrak{D}_{n}$ of $n \times n$ matrices with entries in $\mathfrak{D}$, where $\mathfrak{D}$ is associative, or $\mathfrak{A}=\mathfrak{H}\left(\mathfrak{D}_{n}, \gamma\right)$ is the algebra of selfadjoint elements of $\mathfrak{D}_{n}$ relative to a canonical involution determined by $\gamma$, where $\mathfrak{D}$ is associative if $n>3$ and in any case is an alternative algebra with involution whose self-adjoint elements are in the nucleus.

Proof. If the indicator is $\phi=\frac{1}{4}$ then $\mathfrak{A}$ is commutative by Lemma 5, and hence it is known $[6$, p. 1158$]$ that $\mathfrak{U}=\mathfrak{H}\left(\mathfrak{D}_{n}, \gamma\right)$ as above.

If $\phi=\lambda(1-\lambda) \neq \frac{1}{4}$ then $\lambda \neq \frac{1}{2}$ has an inverse $\mu$ in $\tilde{\Phi}$. Let $\tilde{\mathfrak{U}}=\mathfrak{U}^{(\mu)}$; the $\left\{e_{i}\right\}$ remain idempotents in $\tilde{\mathfrak{A}}$, and since $\widetilde{L}_{e}+\widetilde{R}_{e}=L_{e}+R_{e}$ the Peirce decompositions remain in the same: $\tilde{\mathfrak{I}}_{i j}=\mathfrak{A}_{i j}$. If $x \in \mathfrak{A}_{1}^{[\sigma]}(e)$ then $e \cdot{ }_{\tau} x=\tau e \cdot x+(1-\tau) x \cdot e=$ $=\{\tau \sigma+(1-\tau)(1-\sigma)\} x=\{\tau \odot \sigma\} x$. Since $\mu \odot \lambda=1, \mu \odot(1-\lambda)=0$ we see $\mathfrak{A}_{1}^{[\lambda]}=\tilde{\mathfrak{A}}_{1}^{[1]}, \mathfrak{\mathfrak { A }}_{1}^{[1-\lambda]}=\tilde{\mathfrak{A}}_{1}^{[0]}$, and so from (13) $\tilde{\mathfrak{A}}_{i j}=\mathfrak{E}_{i j}^{[\phi]}=\tilde{\mathfrak{E}}_{i j}^{[0]}$. By assumption there are $u_{i j}, v_{i j} \in \tilde{\mathbb{E}}_{i j}^{[0]}$ with $u_{i j}{ }_{\mu} v_{i j}=u_{i j} \cdot v_{i j}=v_{i j} \cdot u_{i j}=e_{i}+e_{j}$, so $e_{i}$ and $e_{j}$ are connected in $\tilde{\mathfrak{U}}$. By Lemma $6 \tilde{\mathfrak{U}}$ is associative. Denoting ${ }_{\mu}$ by juxtaposition (parentheses are unnecessary by associativity) we set $e_{i j}=u_{1 i} e_{1} v_{1 j}$ (where $\left.u_{11}=v_{11}=e_{1}\right)$. Then $e_{i j} e_{k l}=u_{1 i} e_{1} v_{1 j} u_{1 k} e_{1} v_{1 l}=\delta_{j k} u_{1 i} e_{1} v_{1 l}=\delta_{j k} e_{i l}$ since (8)* shows $e_{1} v_{1 j} u_{1 k} e_{1}=v_{1 j} e_{j} e_{k} u_{1 k}=\delta_{j k} v_{1 j} e_{j} u_{1 j}=\delta_{j k} e_{1} v_{1 j} u_{1 j}=\delta_{j k} e_{1}\left(v_{1 j} u_{1 j}=e_{1}+e_{j}\right.$ if $\left.1 \neq j, v_{11} u_{11}=e_{1}\right)$. Thus $e_{i j}$ are matrix units, and this implies $\tilde{\mathfrak{A}}=\mathfrak{D}_{n}$ for $\mathfrak{D}$ associative. By (17) we obtain $\mathfrak{A}=\left\{\mathfrak{A}^{(\mu)}\right\}^{(\lambda)}=\tilde{\mathfrak{A}}^{(\lambda)}=\mathfrak{D}_{n}^{(\lambda)}$.

5. Structure theory. Throughout this section we will be concerned with finitedimensional algebras. A finite-dimensional noncommutative Jordan algebra is simple it it has no proper ideals and is not a nil algebra, and is semisimple if it is nonzero and has no nonzero nil ideals; the radical is the maximal nil ideal.

THEOREM 2. If $\mathfrak{A}$ is a finite-dimensional noncommutative Jordan algebra, $\mathfrak{R}$ its radical, then $\mathfrak{U} / \mathfrak{R}$ is zero or is semisimple. Any semisimple algebra has an identity and is a direct sum of simple ideals.

Proof. Clearly $\mathfrak{A} / \mathfrak{R}$ has no nonzero nil ideals, so is zero or semisimple. We now suppose $\mathfrak{A}$ is semisimple.

Let $e \neq 0$ be a principal idempotent for a minimal ideal $\mathfrak{I} \subset \mathfrak{A}$ (such exist by finite-dimensionality and the fact that $\mathfrak{I}$ is not nil). By the Corollary to Lemma 2 , 
$\mathfrak{B}=\mathfrak{R}_{0}+\mathfrak{A}_{1}+\mathfrak{R}_{2}$ is an ideal in $\mathfrak{A}$. Now $e \in \mathfrak{I}$ implies $\mathfrak{A}_{1}=\left(L_{e}+R_{e}\right) \mathfrak{A}_{1} \subset \mathfrak{I}$, so $\mathfrak{N}_{i}=P_{i}\left\{\mathfrak{H}_{1}^{2}\right\} \subset P_{i}\{\mathfrak{I}\} \subset \mathfrak{I}$, and thus $\mathfrak{B} \subset \mathfrak{I}$.

We claim $\mathfrak{B}=0$. Suppose not; then by minimality $\mathfrak{B} \subset \mathfrak{I} \Rightarrow \mathfrak{B}=\mathfrak{J}$, and thus $e \in \mathfrak{B}$. Let $\tilde{\mathfrak{B}}=\mathfrak{B}_{\Omega}$ where $\Omega$ is the algebraic closure of $\Phi$. It is standard that $e=\sum_{1}^{n} e_{i}$ is a sum of orthogonal primitive idempotents in $\tilde{\mathfrak{B}}$ (i.e., for $i \neq 0$ in (8) $e_{i}$ is the only nonzero idempotent in $\tilde{\mathfrak{B}}_{i i}$; this implies $\tilde{\mathfrak{B}}_{i i}=\Omega e_{i}+\tilde{\mathcal{B}}_{i i}$ where $\tilde{\mathfrak{Z}}_{i i}^{+}$is a nil subalgebra $\left[8\right.$, p. 702]). Now $e$ principal $\Rightarrow \mathfrak{A}_{0}$ nil $\Rightarrow \mathfrak{A}_{0}^{+}$-nilpotent $\Rightarrow \tilde{\mathfrak{A}}_{0}^{+}$nilpotent $\Rightarrow \tilde{\mathfrak{A}}_{0}$ nil (nil and nilpotent being the same for finite-dimensional commutative Jordan algebras [3, p. 553]) so $\tilde{\mathfrak{B}}_{00}$ is nil. It is well known [1] that if $x \in \tilde{\mathfrak{B}}_{i 0}$ then $P_{00}\left\{x^{2}\right\}$ nil implies $P_{i i}\left\{x^{2}\right\}$ nil $\left({ }^{2}\right)$, hence $P_{i i}\left\{x^{2}\right\} \subset \tilde{\mathbb{B}}_{i i}$. By (11) these span $P_{i i}\left\{\tilde{\mathfrak{B}}_{i 0}^{2}\right)$, so $P_{i i}\left\{\tilde{\mathfrak{B}}_{i 0}^{2}\right\} \subset \tilde{\mathfrak{B}}_{i i}$. Then $e_{i}=P_{i i}(e) \in P_{i i}\{\tilde{\mathfrak{B}}\} \subset P_{i i}\left\{\tilde{\mathfrak{N}}_{2}\right\}$ $=P_{i i}\left\{\tilde{\mathfrak{A}}_{1}^{2}\right\} \subset P_{i i}\left\{\tilde{\mathfrak{B}}_{i 0}^{2}\right\} \subset \tilde{\mathfrak{Z}}_{i i}$ (since $\tilde{\mathfrak{A}}_{1}=\tilde{\mathfrak{B}}_{1}=\Sigma \tilde{\mathfrak{B}}_{i 0}$ ) which is a contradiction.

From $\mathfrak{B}=0$ we conclude $\mathfrak{A}_{1}=0, \mathfrak{A}=\mathfrak{A}_{0} \oplus \mathfrak{A}_{2} ;$ thus $\mathfrak{A}_{0}, \mathfrak{A}_{2}$ are ideals, and by minimality $\mathfrak{A}_{2}=e \mathfrak{A}_{2} \subset \mathfrak{I}$ implies $\mathfrak{I}=\mathfrak{A}_{2}$ has an identity $e$. Ideals in $\mathfrak{I}=\mathfrak{A}_{2}$ and $\mathfrak{R}=\mathfrak{A}_{0}$ are ideals in $\mathfrak{A}$, so $\mathfrak{I}$ is simple and $\mathfrak{A}$ is semisimple. Repeating the procedure with $\mathfrak{K}$ we finally get $\mathfrak{A}=\bigoplus \mathfrak{I}_{i}$ as a direct sum of simple ideals with identities $1_{i}$; then $1=\bigoplus 1_{i}$ is the identity of $\mathfrak{A}$.

THEOREM 3. A finite-dimensional central simple noncommutative Jordan algebra is either

(a) of degree 1,

(b) of degree 2,

(c) a quasi-associative algebra,

(d) a commutative Jordan algebra.

Proof. Since $\mathfrak{A}$ is central, it is well known that $\mathfrak{A}_{\Omega}$ is also simple for $\Omega$ the algebraic closure of $\Phi$. It is clearly enough to prove $\mathfrak{A}_{\Omega}$ is of type (a)-(d), so we may as well assume $\Phi$ algebraically closed from the start.

By Theorem 2, $\mathfrak{A}$ has an identity 1 ; since $\Phi$ is algebraically closed, $1=\sum e$ can be written as a sum of $n$ primitive orthogonal idempotents. If $n \leqq 2$ we have the case (a) or (b). From now on we suppose $n \geqq 3$. By Theorem 1 we need only show the $e_{i}$ are connected.

Taking $e=e_{k}$ in the Corollary to Lemma 2 we see $\mathfrak{B}=\mathfrak{R}_{0}+\mathfrak{A}_{1}+\mathfrak{N}_{2}$ is an ideal; by simplicity $\mathfrak{B}=\mathfrak{A}\left(\mathfrak{B}=0\right.$ would imply $\mathfrak{A}_{1}=0, \mathfrak{A}=\mathfrak{A}_{0} \oplus \mathfrak{A}_{2}$ would have two proper ideals). From this we derive (14), (15), (16), (14)*, (15)* as in Lemma 4.

Let $\phi \in \Phi$. For $i \neq j$ set $\mathfrak{B}_{i j}=\mathfrak{E}_{i j}^{[\phi]}, \mathfrak{C}_{i j}=\mathfrak{A}_{i j} \mathfrak{B}_{i j}+\mathfrak{B}_{i j} \mathfrak{A}_{i j}, \mathfrak{B}_{i i}=\sum_{j \neq i} P_{i i}\left\{\mathfrak{C}_{i j}\right\}$, and $\mathfrak{B}=\bigoplus \mathfrak{B}_{i j}$. We claim $\mathfrak{B}$ is an ideal. If $i \neq j, \mathfrak{A B}_{i j}+\mathfrak{B}_{i j} \mathfrak{A} \subset \mathfrak{B}$ follows

(2) If not, there is an idempotent $0 \neq f \in \Phi\left[P_{i i}\left(x^{2}\right)^{m}\right]=\Phi\left[P_{i i}\left(x^{2 m}\right)\right]=\Phi\left[x^{2 m}\right]$ (where $m$ is chosen so that $\left.P_{00}\left(x^{2 m}\right)^{9}=P_{00}\left(x^{2}\right)^{m}=0\right)$; then $L_{f}$ commutes with $L_{x}, R_{x}$ and $f x=L_{f} x$ $=L_{f}\left(L_{x}+R_{x}\right) e_{i}=\left(L_{x}+R_{x}\right) L_{f} e_{i}=\left(L_{x}+R_{x}\right) f\left(\right.$ since $\left.f \in \tilde{\mathfrak{B}}_{i i}\right)=2 f x, f x=0, f \Phi[x]=0, f 2=0$ which is a contradiction. 
from Lemma 3, (16), and the de finition of $\mathfrak{B}_{i i}$. To show $\mathfrak{U}_{i i}+\mathfrak{B}_{i i} \mathfrak{A} \subset \mathfrak{B}$ it suffices to show $\mathfrak{A}_{i k} P_{i i}\left\{\mathfrak{C}_{i j}\right\}+P_{i \iota}\left\{\mathfrak{C}_{i j}\right\} \mathfrak{A}_{i k} \subset \mathfrak{B}$. First suppose $j \neq k$. Taking $e=e_{j}$, $\mathfrak{N}_{1}=\mathfrak{E}^{[\phi]}$ in Lemma 2 we see the left side above is contained in $\mathfrak{A}_{0} \mathfrak{N}_{0}+\mathfrak{N}_{0} \mathfrak{A}_{0} \subset \mathfrak{N}_{0}$; since $\mathfrak{N}_{1}=\sum_{l \neq j}\left(\mathfrak{E}_{l j}^{[\phi]}=\sum_{l \neq j} \mathfrak{B}_{l j}\right.$ the previous case gives $\mathfrak{A N}_{1}+\mathfrak{N}_{1} \mathfrak{A} \subset \mathfrak{B}$, $\mathfrak{N}_{0}=P_{0}\left\{\mathfrak{X} \mathfrak{N}_{1}+\mathfrak{N}_{1} \mathfrak{A}\right\} \subset P_{0}\{\mathfrak{B}\} \subset \mathfrak{B}$. Finally, suppose $j=k$. Let $z \in P_{i i}\left\{\mathfrak{C}_{i j}\right\}$, $x \in \mathfrak{A}_{i k}=\mathfrak{A}_{i j}$. By (14) we may assume $x=F_{u} v$ for $u \in \mathfrak{A}_{l j}, v \in \mathfrak{A}_{i l}(l \neq i, j$ exists since $n \geqq 3), F=R, L$. But $z \in \mathfrak{A}_{2}\left(e_{i}\right), u \in \mathfrak{A}_{0}\left(e_{i}\right)$ so putting $z, u, e_{i}$ into (2) shows $\left[E_{z}, F_{u}\right]=0$. Then $E_{z} x=E_{z} F_{u} v=F_{u} E_{z} v$; by the case $j \neq k$ we know $E_{z} v \in \mathfrak{B}_{i l}$, so $F_{u} E_{z} v \in \mathfrak{A} \mathfrak{B}_{i l}+\mathfrak{B}_{i l} \mathfrak{A} \subset \mathfrak{B}$ by the first case considered, and thus $E_{z} x \in \mathfrak{B}$ for $E=R, L$. This completes the proof that $\mathfrak{A B}_{i i}+\mathfrak{B}_{i i} \mathfrak{A} \subset \mathfrak{B}$. Hence $\mathfrak{B}$ is an ideal. Since $\Phi$ is algebraically closed $L_{\boldsymbol{e}_{i}}$ has a nonzero eigenvector in $\mathfrak{A}_{i k}$, so we may find $\phi$ with $\mathfrak{E}_{i k}^{[\phi]} \neq 0$, and thus the corresponding $\mathfrak{B}$ is nonzero. By simplicity $\mathfrak{B}=\mathfrak{A}$, and we have shown $\mathfrak{U}_{i j}=\mathfrak{B}_{i j}=\mathfrak{E}_{i j}^{[\phi]}$.

As we remarked in Theorem $2, \mathfrak{A}_{i i}=\Phi e_{i}+\mathcal{Z}_{i i}$ for $\mathfrak{Z}_{i i}$ a nil subspace. $\mathfrak{A}_{i i}$ is spanned by the $P_{i i}\left\{u^{2}\right\}$ for $u \in \mathfrak{A}_{i k}$ by (15), so there exists a $u$ with $u^{2}=\alpha e_{i}+z_{i i}+\beta e_{k}+z_{k k}$ and $\alpha \neq 0$. This implies (see footnote 2) that $\beta \neq 0$, so $u^{2}$ is regular in $\mathfrak{A}_{i i}+\mathfrak{A}_{k k}$, hence $u \in \mathfrak{A}_{i k}=\mathfrak{E}_{i k}^{[\phi]}$ is regular. Thus $e_{i}$ and $e_{k}$ are connected, and we can apply Theorem 1.

Theorems 2 and 3 are due to R. H. Oehmke [9, p. 229] for characteristic different from 3 . It should be noted that he proved Theorem 2 for the much more general case of flexible power-associative algebras.

6. Birepresentations. A birepresentation of the noncommutative Jordan algebra $\mathfrak{A}$ on a bimodule $\mathfrak{M}$ is a pair $(L, R)$ of linear maps $a \rightarrow L_{a}, a \rightarrow R_{a}$ of $\mathfrak{A}$ into the algebra $\mathscr{L}(\mathfrak{M}, \mathfrak{M})$ of linear transformations on the vector space $\mathfrak{M}$ which turns the split null extension $\mathfrak{E}=\mathfrak{A} \oplus \mathfrak{M}$ into a noncommutative Jordan algebra (multiplication in $\mathfrak{E}$ is defined by $a \cdot b=a b, a \cdot m=L_{a} m, m \cdot a=R_{a} m, m \cdot n=0$ for $a, b \in \mathfrak{A}$ and $m, n \in \mathfrak{M})$. Explicitly, the conditions on $(L, R)$ are

(a) $\left[L_{a}, R_{a}\right]=0$,

(b) $L_{a b}-L_{a} L_{b}=R_{b a}-R_{a} R_{b}$,

(c) $\left[L_{a}, R_{a^{2}}\right]=0$,

(d) $L_{a} L_{b}\left(L_{a}+R_{a}\right)+R_{b a^{2}}=R_{a^{2}} R_{b}+L_{a b}\left(L_{a}+R_{a}\right)$.

Clearly these are necessary by (3), (4). It is well known $[10$, p. 473] that $\mathbb{E}$ is a noncommutative Jordan algebra if

$$
\left[L_{x}, R_{x}\right]=0=\left[L_{x}, R_{x^{2}}\right]
$$

for all $x \in \mathfrak{E}$. Writing $x=a+m$ and expanding these relations, the terms involving only $a$ 's vanish on $\mathfrak{A}$ by definition of noncommutative Jordan algebra and on $\mathfrak{M}$ by (a),(c); the terms involving two or more $m$ 's vanish since $\mathfrak{M}$ is an ideal in $\mathfrak{E}$ with $\mathfrak{M}^{2}=0$; for the same reason the terms involving one $m$ vanish on $\mathfrak{M}$, and they vanish on all $b \in \mathfrak{A}$ by (b),(d). Thus, the conditions are sufficient. 
Suppose $\mathfrak{A}$ has an identity 1 and $\mathfrak{M}$ is a bimodule for $\mathfrak{U}$; by (5) we have a Peirce decomposition

$$
\mathfrak{E}=\mathfrak{E}_{0} \oplus \mathfrak{E}_{1} \oplus \mathfrak{E}_{2}
$$

relative to 1 . Since $\mathfrak{M}$ is an ideal in $\mathfrak{E}$ we have

$$
\mathfrak{M}=\mathfrak{M}_{0} \oplus \mathfrak{M}_{1} \oplus \mathfrak{M}_{2}
$$

for $\mathfrak{M}_{i}=P_{i}\{\mathfrak{M}\} \subset \mathfrak{E}_{i}$. Notice that by (6) $\mathfrak{A} \subset \mathfrak{E}_{2}$ shows the $\mathfrak{M}_{i}$ are sub-bimodules of $\mathfrak{M}$. We have $L_{a}=R_{a}=0$ on $\mathfrak{M}_{0} \subset \mathfrak{E}_{0}$ for $a \in \mathfrak{A} \subset \mathfrak{E}_{2}$ by (6), so $L=R=0$ on $\mathfrak{M}_{0} ; \mathfrak{M}_{0}$ is a zero bimodule. On $\mathfrak{M}_{2}$ we have $R_{1}=L_{1}=I$, so $\mathfrak{M}_{2}$ is a iunital bimodule. $\mathfrak{M}_{1}$ will be called a special bimodule even though in general little can be said about its structure; we have $R_{1}+L_{1}=I$ on $\mathfrak{M}_{1}$.

An $\mathfrak{A}$-bimodule $\mathfrak{M}$ is irreducible if it is not the zero bimodule and has no proper subbimodules, and is completely reducible if it is a direct sum of irreducible bimodules.

7. Pathologies. Here we will show that for algebras of degree 1 or 2 the usual structure theorems for bimodules fail to hold.

EXAMPLE $1 . \mathfrak{U}=\Phi 1, L_{1}=T, R_{1}=I-T$ where $T$ is an arbitrary linear transformation on a (perhaps infinite-dimensional) space $\mathfrak{X}$. (a) and (c) are trivial, (b) follows from $L_{1 \cdot 1}-L_{1} L_{1}=T-T^{2}=(I-T)-(I-T)^{2}=R_{1 \cdot 1}-R_{1} R_{1}$, and (d) from $L_{1} L_{1}\left(L_{1}+R_{1}\right)+R_{1 \cdot 1 \cdot 1}=T^{2}+(I-T)=(I-T)^{2}+T=R_{1 \cdot 1} R_{1}+L_{1 \cdot 1}\left(L_{1}+R_{1}\right)$. Thus $(L, R)$ gives a special birepresentation of $\mathfrak{A}$ on $\mathfrak{X}$.

Naturally all unital $\mathfrak{A}$-bimodules are completely reducible and all irreducible birepresentations are finite-dimensional, but the above special bimodule need be completely reducible and the enveloping associative algebra of the birepresentation need not be finite-dimensional. From this example we can construct unital birepresentations of the semisimple algebra $\Phi e_{1} \oplus \Phi e_{2}$ which are not completely reducible by setting $L_{e_{1}}=R_{e_{2}}=T, R_{e_{1}}=L_{e_{2}}=I-T$. There are infinitely many nonisomorphic irreducible finite-dimensional special $\mathfrak{A}$-bimodules, one for each irreducible polynomial $p(x)$ in $\Phi[x]$ (take $T$ to be cyclic with $p(x)$ as its minimum polynomial).

Example 2. $\mathfrak{A}=\Phi 1+\mathfrak{M}$ is the Jordan algebra of degree 2 defined by a bilinear form $(m, n)$ on $\mathfrak{M}$. If $\left\{m_{i}\right\}$ is a basis for $\mathfrak{M}$, set $L_{1}=R_{1}=I, L_{m_{i}}=-R_{m_{i}}=T$ where again the $T_{i}$ are any linear transformations on $\mathfrak{X}$. The symmetrized birepresentation $L_{a}^{+}=\left(L_{a}+R_{a}\right) / 2$ is thus just the trace birepresentation $a \rightarrow(\operatorname{tr} a) I$, and this is always a (commutative) Jordan birepresentation. Now it is standard $[10$, p. 473$]$ that if $\mathfrak{E}$ is flexible and $\mathfrak{E}^{+}$is Jordan then $\mathfrak{E}$ is a noncommutative Jordan algebra; we have just seen $\mathbb{E}^{+}$is Jordan, so it only remains to check flexibility. (a) is trivial since for $a=\lambda 1+m, R_{a}=2 \lambda I-L_{a}$; (b) is trivial if $a$ or $b$ is 1 , and if $a=m_{i}, b=m_{j}$ then $L_{a b}-L_{a} L_{b}=\left(m_{\imath}, m_{j}\right) I-T_{i} T_{j}$ $=\left(m_{j}, m_{i}\right) I-\left(-T_{i}\right)\left(-T_{j}\right)=R_{b a}-R_{a} R_{b}$. 
Hence we have constructed a unital birepresentation which again need not be completely reducible or have finite-dimensional enveloping algebra. But it is even worse than that. Assuming the dimension of $\mathfrak{M}$ to be greater than 1 , let $\mathfrak{X}$ have basis $\left\{x_{n}\right\}_{n \in z}$ and take $T_{1}=S, T_{2}=S_{m}, T_{i}=0(i>2)$ where $S$ is the shift operator $S x_{n}=x_{n+1}$ and where $S_{m} x_{n}=0$ (if $m$ does not divide $n$ ), $S_{m} x_{k m}=x_{-k}$. One easily verifies that $S_{m}=S S_{m} S^{m}$, and thus the birepresentations for different choices of $m$ are nonisomorphic. It is not hard to check that for $m \geqq 2$ the bimodules are irreducible, and hence we have found infinitely many nonisomorphic infinite-dimensional irreducible unital $\mathfrak{A}$-bimodules.

8. Representation theory. The pathologies of the last section show that we cannot hope to prove complete reducibility of representations involving algebras of degree 1 or 2 , so we content ourselves with

THEOREM 4. If $\mathfrak{A}=\bigoplus \mathfrak{A}_{i}$ is a finite-dimensional separable noncommutative Jordan algebra whose simple summands $\mathfrak{A}_{i}$ are of degree $\geqq 3$ then every $\mathfrak{A}$-bimodule is completely reducible.

Proof. By separability it suffices to assume $\Phi$ is algebraically closed. Suppose $\mathfrak{B}$ is any simple noncommutative Jordan algebra over $\Phi$ with identity $1=\sum e_{i}$ which is the sum of $n \geqq 3$ connected idempotents, and let $\mathfrak{N}$ be a special $\mathfrak{B}$-bimodule. Relative to $\left\{e_{i}\right\}$ the split null extension $\mathfrak{F}=\mathfrak{B} \oplus \mathfrak{N}$ has the Peirce decomposition $\mathfrak{F}=\bigoplus \mathfrak{F}_{i j}$ by (8), and since $\mathfrak{N}$ is special it has the Peirce decomposition $\mathfrak{N}=\bigoplus \mathfrak{N}_{i 0}$. By connectivity, for $i, j, 0 \neq$ there are $u_{i j}, v_{i j} \in \mathfrak{E}_{i j}^{[\phi]}$ with $u_{i j} v_{i j}=e_{i}+e_{j}$ (where $\phi=\lambda(1-\lambda)$ is the indicator of $\mathfrak{B}: \lambda \in \Phi$ since $\Phi$ algebraically closed). Then $\mathfrak{N}_{i 0}=\left(u_{i j} v_{i j}\right) \mathfrak{N}_{i 0}+\mathfrak{N}_{i 0}\left(u_{i j} v_{i j}\right) \subset \mathfrak{N}_{i 0}^{[\phi]}$ by (12) and Lemma 3 , hence

$$
\mathfrak{N}=\mathfrak{C}_{1}^{[\phi]}(1)=\mathfrak{N}^{[\lambda]}(1)+\mathfrak{N}^{[1-\lambda]}(1)
$$

since $\mathfrak{B}$ is contained in the Peirce space $\mathfrak{F}_{2}(1)$ relative to 1 , Lemma 3 shows the $\mathfrak{N}^{[\mu]}(1)$ are sub-bimodules.

Now suppose $\mathfrak{M}$ is an $\mathfrak{X}$-bimodule, where $\mathfrak{A}$ is as in the statement of the theorem. Applying the Peirce decomposition (8) relative to the idempotent $1=\sum 1_{i}$ to the split null extension $\mathfrak{E}=\mathfrak{A} \oplus \mathfrak{M}$ (where $1_{i}$ is the identity of $\mathfrak{U}_{i}$ - note that 1 need not be the identity of $(\mathbb{E})$ we get

$$
\begin{gathered}
\mathbb{E}=\bigoplus_{i, j=0}^{n} \mathfrak{E}_{i j}, \\
\mathfrak{M}=\bigoplus_{i, j=0}^{n} \mathfrak{M}_{i j} .
\end{gathered}
$$

Since $\mathfrak{A}_{i} \subset \mathfrak{E}_{i i}$ we see by (9) that each $\mathfrak{M}_{i j}$ is a sub- $\mathfrak{U}$-bimodule, so it suffices to prove each $\mathfrak{M}_{i j}$ is completely reducible. 
$\mathfrak{M}_{00}$ is a zero bimodule, hence trivially completely reducible (with the irreducible submodules being the one-dimensional subspaces).

$\mathfrak{M}_{i 0}$ is a special $\mathfrak{A}_{i}$-bimodule (i.e. the other $\mathfrak{U}_{j}$ act trivially). By (18) we see $\mathfrak{M}_{i 0}=\mathfrak{M}_{i 0}^{[\lambda]}+\mathfrak{M}_{i 0}^{[1-\lambda]}$, so it will suffice if $\mathfrak{M}_{i 0}^{[\lambda]}$ is completely reducible. If the indicator of $\mathfrak{U}_{i}$ is $\phi=\frac{1}{4}$ then $\lambda=1-\lambda=\frac{1}{2}$; thus $L_{1_{i}}=R_{1_{i}}=\frac{1}{2} I$ on $\mathfrak{M}_{i 0}^{[1 / 2]}$, so for $\quad x \in \mathfrak{A}_{i}, \quad L_{x}-R_{x}=L_{x .1_{i}}-R_{1_{i} . x}=L_{x} L_{1_{i}}-R_{x} R_{1_{i}} \quad($ by $\quad(3))=\frac{1}{2}\left(L_{x}-R_{x}\right)$, hence $L_{x}=R_{x}$ and we have a special commutative Jordan bimodule. This is known to be completely reducible [7,p.69]. If $\phi \neq \frac{1}{4}$ we may mutate $\mathfrak{A}_{i}$ so that it becomes associative; ideals in $\mathfrak{E}_{i}=\mathfrak{A}_{i} \oplus \mathfrak{M}_{i 0}^{[\lambda]}$ coincide with those in its $\mu$-mutation $\left(\mu \neq \frac{1}{2}\right)$, so it suffices to consider the case where $\mathfrak{A}_{i}$ is associative and $\lambda=1$. Then for $x \in \mathfrak{A}_{i}$ we have $L_{x}-R_{x}=L_{x .1_{i}}-R_{1_{i} \cdot x}=L_{x} L_{1_{i}}-R_{x} R_{1_{i}}$ (by (3)) $=L_{x}$ (since $L_{1_{i}}=I, R_{1_{i}}=0$ on $\mathfrak{M}_{i 0}^{[1]}$ ). Thus $R_{x}=0$, and from (3) $L_{x y}=L_{x} L_{y}$. Thus we have an ordinary left module for the simple associative algebra $\mathfrak{A}_{i}$, and this is obviously completely reducible.

$\mathfrak{M}_{i i}$ is a unital $\mathfrak{A}_{i}$-bimodule; since $\mathfrak{U}_{i}$ has $n \geqq 3$ connected idempotents so does $\mathfrak{E}_{i}=\mathfrak{A}_{i} \oplus \mathfrak{M}_{i i}$. If the indicator of $\mathfrak{A}_{i}$ is $\phi=\frac{1}{4}$ then $\mathfrak{E}_{i}$ is commutative by Lemma 5 , and $\mathfrak{M}_{i i}$ is completely reducible as a unital commutative Jordan bimodule for the Jordan algebra $\mathfrak{A}_{i}\left[7\right.$, p. 69]. If $\phi \neq \frac{1}{4}$ we may again perform a mutation which reduces us to the case $\phi=0$; then $\mathfrak{E}_{i}$ is associative by Lemma 6 , and $\mathfrak{M}_{i i}$ is completely reducible as a unital associative bimodule for the associative algebra $\mathfrak{A}_{i i}[7$, p. 61].

Finally, consider $\mathfrak{M}_{i j}(i, j, 0 \neq)$. This is special as an $\mathfrak{A}_{i}$ or $\mathfrak{A}_{j}$ bimodule, so by (18) we have $\mathfrak{M}_{i j}=\mathfrak{M}_{i j}^{\left[\phi_{i}\right]}=\mathfrak{M}_{i j}^{\left[\phi_{j}\right]}$ where $\phi_{i}, \phi_{j}$ are the indicators of $\mathfrak{A}_{i}, \mathfrak{U}_{j}$. Thus $\mathfrak{I}_{i}$ and $\mathfrak{U}_{j}$ must have the same indicator $\phi$. If $\phi=\frac{1}{4}$, then as before $\mathfrak{M}_{i j}$ is a special Jordan bimodule for the Jordan algebras $\mathfrak{A}_{i}, \mathfrak{A}_{j}$, hence it is completely reducible $[17$, p. 69$]$. The case $\phi \neq \frac{1}{4}$ may be reduced to the case $\phi=0$, so we may assume $\mathfrak{A}_{i}, \mathfrak{A}_{j}$ are associative. By (18) it is enough if the sub-bimodules $\mathfrak{M}_{i j}^{[1]}\left(1_{i}\right)=\mathfrak{M}_{i j}^{[0]}\left(1_{j}\right)$ and $\mathfrak{M}_{i j}^{[0]}\left(1_{i}\right)=\mathfrak{M}_{i j}^{[1]}\left(1_{j}\right)$ are completely reducible. As before, on the first one we have $L_{x y}=L_{x} L_{y}, R_{x}=0$ for $x, y \in \mathfrak{U}_{i}, R_{y x}=R_{x} R_{y}, L_{x}=0$ for $x, y \in \mathfrak{A}_{j}$; hence it is a left module for the simple associative algebras $\mathfrak{A}_{i}$ and $\mathfrak{U}_{j}^{\prime}$ (where $\mathfrak{U}_{j}^{\prime}$ is anti-isomorphic to $\mathfrak{A}_{j}$ ). By (3) we have $\left[L_{x}, L_{y^{\prime}}\right]=\left[L_{x}, R_{y}\right]$ $=\left[R_{x}, L_{y}\right]=[0,0]=0$ if $x \in \mathfrak{A}_{i}$ and $y^{\prime} \in \mathfrak{A}_{j}^{\prime}$ corresponds to $y \in \mathfrak{U}_{j}$, so the representations commute, and we have a unital left module for the simple associative algebra $\mathfrak{A}_{j} \otimes \mathfrak{A}_{j}^{\prime}$ (remember $\Phi$ is algebraically closed, so $\mathfrak{A}_{i}, \mathfrak{A}_{j}$ are central). Thus $\mathfrak{M}_{i j}^{[1]}\left(1_{i}\right)$ is completely reducible, and similarly for $\mathfrak{M}_{i j}^{[0]}\left(1_{i}\right)$.

Notice that the proof allows us to determine the irreducible bimodules by using the results of [7] and [6].

\section{REFERENCES}

1. A. A. Albert, Power-associative rings, Trans. Amer. Math. Soc. 64 (1948), 552-593.

2. - A theory of trace-admissable algebras, Proc. Nat. Acad. Sci. U.S.A. 35 (1949), 317-322. 
3. ——, A structure theory for Jordan algebras, Ann. of Math. (2) 48 (1947), 546-567.

4. S. Eilenberg, Extensions of general algebras, Ann. Soc. Polon. Math. 21 (1948), 125-134.

5. N. Jacobson, A theorem on the structure of Jordan algebras, Proc. Nat. Acad. Sci. U.S.A. 42 (1956), 140-147.

6. - A coordinatization theorem for Jordan algebras, Proc. Nat. Acad. Sci. U.S.A. 48 (1962), 1154-1160.

7. __ Structure of alternative and Jordan bimodules, Osaka Math. J. 6 (1954), 1-71.

8. K. McCrimmon, Jordan algebras of degree 1, Bull. Amer. Math. Soc. 70 (1964), 702.

9. R. H. Oehmke, On flexible algebras, Ann. of Math. (2) 68 (1958), 221-230.

10. R. D. Schafer, Noncommutative Jordan algebras, Proc. Amer. Math. Soc. 6 (1955), $472-475$.

11. K. McCrimmon, Norms and noncommutative Jordan algebras, Pacific J. Math. 15 (1965), 1-32.

Massachusetts Institute of Technology,

Cambridge, Massachusetts 